\title{
Small-angle X-ray scattering beamlines at the photon factory
}

\author{
N. Shimizu, H. Takagi, Y. Nagatani, K. Yonezawa, T. Mori, K. Yatabe, M. Takahashi, K. Oyama, N. Igarashi \\ Institute of Materials Structure Science, High Energy Accelerator Research Organization (KEK), Tsukuba, Ibaraki, Japan \\ nobutaka.shimizu@kek.jp
}

Three small-angle X-ray scattering (SAXS) beamlines, BL-6A, BL-10C, and BL-15A2, are in operation at the Photon Factory, a synchrotron radiation facility in Japan, and are commonly utilized for versatile application to carry out the structural analysis and the structure-property correlation studies for soft and hard materials including biological macromolecules. The light source of BL-6A is a bending magnet, and the available X-ray energy is fixed at $8.3 \mathrm{keV}(1.5 \AA)$. The maximum camera length is $2.5 \mathrm{~m}$, and SAXS/WAXS (wide-angle X-ray scattering) measurements are performed using PILATUS3 1M (Dectris) for SAXS and PILATUS 100K for WAXS as detectors, respectively. BL-10C is also the bending magnet beamline, and the available X-ray energy range is generally 7.0-14.0 $\mathrm{keV}(0.89-1.77 \AA)$. The Max. camera length is $3.0 \mathrm{~m}$, and SAXS/WAXS measurements can be performed with PILATUS3 $2 \mathrm{M}$ for SAXS and PILATUS3 200K for WAXS. BL-15A is the short-period undulator beamline, and 15A1 and 15A2 are dedicated to XAFS and SAXS, respectively. BL-15A2 has two dedicated diffractometers, one for hard X-rays (5.7-15 keV, Max. camera length: $3.5 \mathrm{~m})$ and the other for tender X-rays (2.1-5.4 keV, Max. camera length: $0.8 \mathrm{~m}$ ), and these are tandemly installed against the beam in BL15A2. PILATUS3 2M for SAXS and PILATUS3 300K-W for WAXS are installed as detectors. Because this PILATUS3 2M is vacuum compatible, it can be directly connected to the dedicated vacuum diffractometer for tender X-rays use under the vacuum condition. We have also installed a special setting to connect the hard X-ray system to the tender X-ray system. The max. camera length is $6.5 \mathrm{~m}$ at that time, and the SAXS resolution reaches $1500 \mathrm{~nm}$ using $2.1 \mathrm{keV}$. The devices for BioSAXS are installed in BL10C and BL-15A2, which can be used not only for SEC-SAXS but also for titration-SAXS and time-resolved SAXS using microfluidic cells. We will introduce the latest measurement and analysis environment at these beamlines in this presentation.
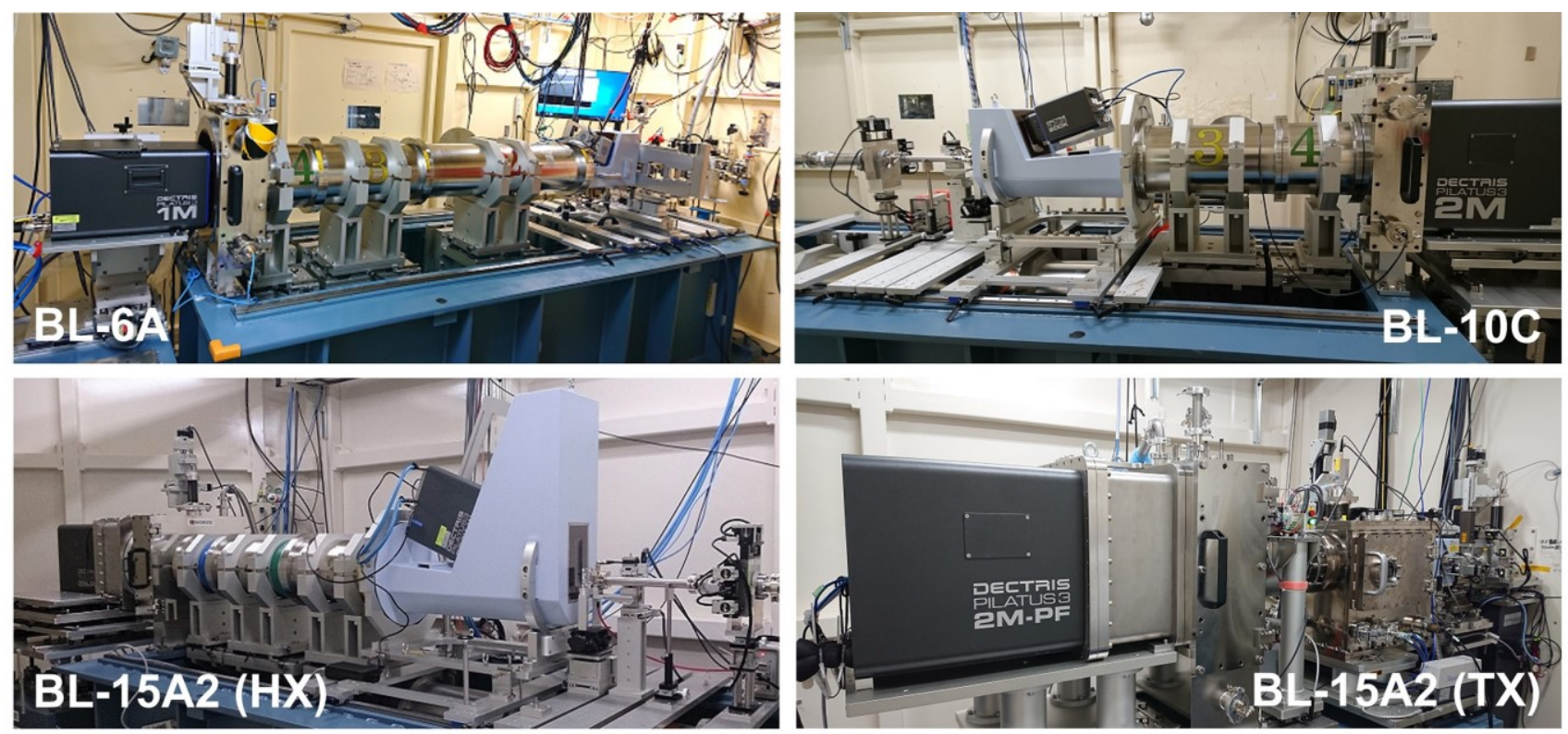

Figure 1. Photos of PF SAXS beamlines.

Keywords: small-angle X-ray scattering; beamline; synchrotron facility 